\title{
Electroencephalogram with Machine Learning for Estimation of Mental Confusion Level
}

\author{
Harsh Kumar, Mayank Sethia, Himanshu Thakur, Ishita Agrawal, Swarnalatha P
}

\begin{abstract}
Estimating the mental state of an individual is crucial to many applications. A quantitative measure of the confusion one faces while doing a task can be useful in determining which subtask is the most difficult. This paper thus aims to develop an algorithm to estimate the confusion score using EEG signals collected using a Neurosky Mindwave Headset. Also, a full contextual audio based confusion score is generated to improve the system's resilience. In this paper, the final algorithm is used to propose an EEG based system to enable the UI/UX testing which can help in confusion estimation and thus provide a qualitative means to measure the attention and concentration level of people which can be extended to various applications. The raw EEG data collected from the device was used to calculate the confusion score using various Machine Learning algorithms. This brain computer interface (BCI) system can be extended for calculating the confusion score of a person which can be used for various applications such as teaching, child health monitoring, suicide prevention, mental health analysis etc. The brain computer interface thus calculates the confusion score and based on the threshold value of the attention and concentration level it performs certain actions such as sending messages and alerts to emergency contacts. This is further extended to solve the problem of Usability testing in Human Computer Interaction.
\end{abstract}

Keywords- Brain Computer Interface, confusion score, attention level, concentration level, mental health, human computer interaction

\section{INTRODUCTION}

Most mental health analysis measures involve talking to a psychiatrist or a doctor, parents talking to their child or a teacher finding out what are the problems that their students are facing but all through direct talk. In most cases, the student or the patient is not very comfortable or is not able to express exactly how he or she feels. This leads to ambiguity and partial representation of data. This we term as a quantitative process where based on the inputs of the student or the patient or any concerned person decisions are made on the understanding of the other person. Through this paper and our project, we aim to solve this issue by building a brain computer interface for qualitative as well as quantitative analysis of a person's mental health.

Revised Manuscript Received on December 30, 2019.

* Correspondence Author

Harsh Kumar, final year B. Tech Computer Science student from the School of Computer Science and Engineering at VIT Vellore.

Mayank Sethia, final year B. Tech Computer Science student from the School of Computer Science and Engineering at VIT Vellore.

Himanshu Thakur, final year B. Tech Computer Science student from the School of Computer Science and Engineering at VIT Vellore.

Ishita Agrawal, final year B. Tech Computer Science student from the School of Computer Science and Engineering at VIT Vellore.

Swarnalatha P, Associate Professor at the School of Computer Science and Engineering at VIT Vellore.

(C) The Authors. Published by Blue Eyes Intelligence Engineering and Sciences Publication (BEIESP). This is an open access article under the CC BY-NC-ND license (http://creativecommons.org/licenses/by-nc-nd/4.0/)
Our brain computer interface mainly involves a hardware device which is to be put up over the head of the person, a software dashboard for analysis, our unique algorithm for the processing of data and a supporting application in times of emergency. We try to measure a person's attention, meditation and concentration levels by analyzing his EEG signals. The dashboard helps to provide inputs to the student or patient and also shows the output to the administrator. In times when the emotional states are too low or below a particular threshold value, we alert the emergency contacts of the person through the app and make sure that the patient or student receives all immediate support. We have also carried out usability testing on our product to make sure we can improve upon the shortcomings. Usability testing is very important from a business point of view. It determines whether people are able to use your product easily, if they able to identify the features and also generate desirable results from the same. Keeping this is in mind, we tried out our product on a group of students, to improve our product with every iteration. We also tried various algorithms to find out which works the best and gives the highest accuracy. Usability testing is considered to be one of Human Computer Interactions biggest evaluator or impactor. It is highly practiced by design professionals and we have tried to incorporate that into our product. After testing with a lot of algorithms, we found that the Random Forest Classifier was giving the best result. We improved upon the dashboard to not only show the data as texts but also in the graphical form- in pie charts and bar graphs. Both audio and text input features were incorporated keeping in mind the diversity of the students and patients. In Human Computer Interaction there is a stark distinction between the main stakeholder's problem and the problem viewed by the user. Our usability testing helped us gain more insights from the view of the users. We thus calibrated our product accordingly. We realized there could be situations of emergency where a student or a patient could have a very harsh mental state- he or she could feel suicidal, depressed, very anxious, very sad etc. and the need of the hour would be to inform his near and dear ones and for him to have all the tools so that he can reach out for help. From the administrator's point of view, the dashboard would reflect the patient's confusion score which is a result of his attention, meditation, concentration levels etc. which would help for further analysis. The App developed was incorporated with one click panic button, nearby hospitals and police stations, emergency toolkits and an AI chatbot to talk to for various needs- for medical help, when someone is feeling depressed, suicidal or lonely etc. It was built as a perfect guide for a person supporting him or her in times of adversity. 
Characterization of usability testing seems to be more complex and requires effective analysis and involvement as compared to the usability world described by Nielsen's intrinsic view. The brain computer interface coupled with the software designs and algorithms would thus help us to build a qualitative way to measure and improve upon the mental health of a person.

\section{LITERATURE SURVEY}

Published in 2014 in the International Conference on Audio, Language and Image Processing, Shanghai by G. Lv, $\mathrm{S}$. Hu and X. Lu the paper aims at speech based emotion recognition. The author tries out 3 different methods. He starts with a static SVM model, followed by a dynamic Hidden Markov Model(HMM) and goes on to propose a 45dimensional Dynamic Bayesian Network(DBN) which had 3 layers. The 3 layered DBN performed much better than the other 2 algorithms in real-time as it was able to measure the sudden changes in emotion. The HMM although accurate would take a lot of time for training since it had a lot of parameters and hence was not preferred. [11]

Yongbin Gao, Hyo Jong Lee, Raja Majid Mehmood in 2015 proposed in their paper - Deep learning of EEG signals for emotion recognition a deep learning method which would produce better results than the conventional methods. In this approach the deep learning algorithms would be directly applied on the raw signals without carrying out feature extraction on the data. 3 layers of restricted Boltzmann machines(RBMs) were used for pre-training. It was further subjected to back propagation for getting better results subject by subject. The EEG signals of 14 channels are fed as a vector into the algorithm with 4 different emotions as outputs namely - happy, sad, calm and scared. The EEGLAB is used to measure the frequency of the EEG signals. Finally, cross validation is performed to achieve the average results. For each emotion, 45 epochs are run and this process achieves better performance and more accurate results than the conventional algorithms. [17]

In 2016 a paper on EEG-based automatic emotion recognition: Feature extraction, selection and classification methods published by Ackermann P and his colleagues used the DEEP dataset to classify and automatically detect emotions. They stressed the importance of a device coupled with software algorithms that could automatically read the EEG signals from the brain and help them classify based on their frequency into different emotion types. They thus aimed at a physiological means for measuring the mental state of a person using certain devices such as pulse meters etc. [9] Later in 2016 Priyanka S. Ghare and A.N. Paithane in their paper - Human emotion recognition using nonlinear and non-stationary EEG signal proposed a GUI based method for automatic emotional recognition. The algorithm used is SVM, since it provides better result as it has multilayer properties. The raw EEG signals are obtained from the database, which has a lot of noise and thus needs to be cleaned. It is then subjected to an Adaptive Finite Impulse Response (FIR) filter followed by Least Means Square Method(LMS) following which the signals are decomposed into 4 different power spectrums namely alpha, beta, gamma and delta. SVM is then used to classify them into emotions.
Daubechies wavelet function is the function used to extract the features from the raw EEG signals which are then fed into the system. [16]

Published in 2016 in the International Conference on Connected Health by S. Berrouiguet and his team proposed an E-Health Mobile Application based platform which was similar to a text messaging app, where the patient could send his or her problems over the app. This was done keeping in mind that many patients don't feel very comfortable telling everything in front of the doctor. The app was supported with hi-tech security, so that data would not be lost. Various machine learning and data mining algorithms would be used over this dataset which would be kept highly secure. It would also send out recommendations to the patients based on the collected information. Another USP of the system was the Ecological Momentary Assessment (EMA) feature along with a simple user interface and a patient friendly application. [10]

Published in 2017 in International Conference on Innovations in Power and Advanced Computing Technologies by Prashant Lahane, Mythili Thirugnanam the paper showcases the use of Frequency Cepstral Coefficient(FCC) Method to classify human emotions. It uses the DEAP dataset for feature extraction. For the FCC algorithm only the alpha band among the 5 other band are considered since it provides a more stable mental state and gives more accurate results. It also has a more reliable frequency band compared to other bands. The authors also used KNN to classify the emotions into basically 2 typeshappy and sad. While KNN gave an accuracy for $80 \%$, FCC was able to perform much better giving an accuracy of $90 \%$ for the DEAP dataset. [18] .

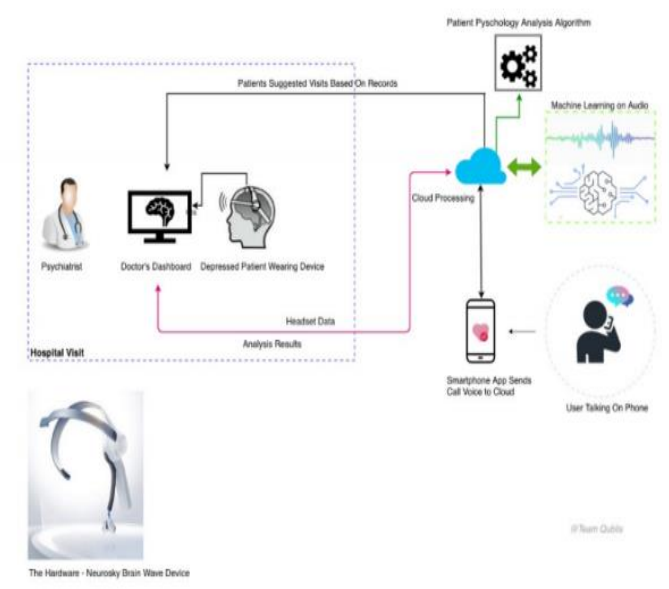

Fig1.0 Main Architecture Diagram for Brain Computer Interface 


\section{ARCHITECTURE}

- Neurosky Mindwave Headset Device - The Neurosky Mindwave device is a special hardware device used to capture the EEG signals from the neurons of the brain. It then classifies these signals into various values such as alpha, beta, gamma, delta, theta which are basically its power values. We then send this data into our algorithm for further processing and to calculate the threshold. The circuit is completed by connecting one part of the device to the ear and the other to the sensor arm.

- Brain Computer Interface - This is the software interface designed for UI/UX testing. It contains a dashboard where the concentration and attention levels of the students are recorded.

- Android App - The Android App is developed with the idea for mobility and to send messages to contacts in case of emergencies, with their real-time location. Other features such as the hospital address along with the contact number, the nearest police station along with their contact number and an offline first-aid kit as well as chatbot is provided for immediate use and help. A basic toolkit and a single-click panic button is also incorporated in case of emergency.

\section{METHODOLOGY}

The Neurosky Mindwave Device helps translate the EEG signals from the neurons to the different power spectrums of the alpha, beta, gamma, delta and theta waves which have different frequencies in the range from $1-100 \mathrm{~Hz}$ as shown in the table below. These different frequencies signify different levels of mental condition, attention and concentration level.

\begin{tabular}{|c|c|c|c|c|}
\hline $\begin{array}{l}\text { S } \\
\text { No. }\end{array}$ & \multicolumn{2}{|c|}{$\begin{array}{l}\text { Power Spectrum } \\
\text { Type(Wave) }\end{array}$} & $\begin{array}{l}\text { Frequenc } \\
\mathrm{y}(\text { in } \mathrm{Hz} \text { ) }\end{array}$ & $\begin{array}{l}\text { Confusion } \quad \text { Score } \\
\text { Analysis }\end{array}$ \\
\hline 1. & \multicolumn{2}{|c|}{ Alpha Wave } & $7.5-12.5$ & $\begin{array}{ll}\text { At peace, } & \text { good } \\
\text { concentration, } & \text { fully } \\
\text { conscious } & \end{array}$ \\
\hline \multirow[t]{3}{*}{2.} & \multirow[t]{3}{*}{$\begin{array}{l}\text { Beta } \\
\text { Wave }\end{array}$} & Low & $13-15$ & $\begin{array}{l}\text { Less concentration, } \\
\text { lesser attention }\end{array}$ \\
\hline & & Medium & $16-20$ & Conscious, aware \\
\hline & & High & $21-30$ & $\begin{array}{l}\text { Highly conscious, } \\
\text { medium to good } \\
\text { concentration. }\end{array}$ \\
\hline 3. & \multicolumn{2}{|c|}{ Gamma Wave } & $31-100$ & $\begin{array}{l}\text { Self and environment } \\
\text { aware, alert }\end{array}$ \\
\hline 4. & \multicolumn{2}{|c|}{ Delta Wave } & $0.1-3.5$ & $\begin{array}{lr}\text { Very little } \\
\text { consciousness, drowsy, } \\
\text { little attention }\end{array}$ \\
\hline 5. & \multicolumn{2}{|c|}{ Theta Wave } & $4-7$ & $\begin{array}{l}\text { Drowsy, very relaxed, } \\
\text { very little consciousness }\end{array}$ \\
\hline
\end{tabular}

Table1. Classification of the power spectrums(waves) and Confusion Score Analysis.
Due to the lack of database, we created our own dataset. EEG signal values and the power spectrums were collected by sampling them over various students during different times and under different conditions. The EEG signals were then split into 5 different power spectrums as shown in the table above, the time stamp was also noted. Voice samples were also collected and stored in a similar way from various students at different external conditions. The implementation was carried out in 5 major steps as shown in the diagram below.

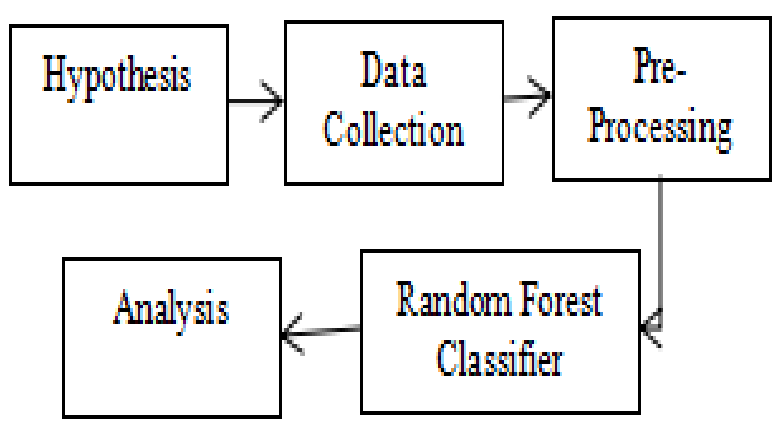

Fig2.0: Synapse- Implementation Interface

After the data collection, it is sent for pre-processing where the noise from the data is removed. The frequencies need to be then sent to an Analog to Digital Converter which has a 12-bit resolution and classifies it into different categories of power spectrums or brain waves. The internal boards of the device then send these brain wave categories via a wireless medium to the system, which then gets displayed on the dashboard.

The algorithm used to do this is the Fast Fourier Transform which helps to classify these brainwaves. The attention, concentration and meditation levels are thus derived which constitute the confusion score. Thus an interface is developed for the brain computer interface. The dashboard thus helps to see the attention, concentration and meditation or relaxed levels of a person and thus helps in qualitative analyses of one's mental state. All these parameters together constitute the confusion score, which is how confused a particular person is during the test.

The different values of the confusion matrix are pushed at Realtime to the Firebase Database. Further computations are done at the database. For confusion scores less than a threshold value, the Realtime location of the concerned person is sent to his emergency contacts and the person is also alerted. The Android Application developed also offers other features useful in times of emergency like one-click panic button, offline first-aid and AI chatbot support, Maps with the nearest police stations and hospitals along with their contact details and an emergency toolkit. 


\section{RESULTS AND ANALYSIS}

\begin{tabular}{|r|r|r|r|r|r|r|r|r|r|r|r|r|r|r|r|}
\hline attention meditation delta & theta & aplha1 & alpha2 & beta1 & beta2 & gamma1 & gamma2 & tstamp \\
\hline 37 & 35 & 466390 & 502169 & 11753 & 213518 & 4889 & 193247 & 2361 & 1128603 & 0 \\
\hline 37 & 35 & 466390 & 502169 & 11753 & 213518 & 4889 & 193247 & 2361 & 1128603 & 0 \\
\hline 37 & 35 & 466390 & 502169 & 11753 & 213518 & 4889 & 193247 & 2361 & 1128603 & 0 \\
\hline 41 & 29 & 858306 & 386602 & 8884 & 287159 & 5455 & 231785 & 1233 & 1122013 & 0 \\
\hline 41 & 29 & 858306 & 386602 & 8884 & 287159 & 5455 & 231785 & 1233 & 1122013 & 0 \\
\hline 41 & 29 & 858306 & 386602 & 8884 & 287159 & 5455 & 231785 & 1233 & 1122013 & 0 \\
\hline 56 & 35 & 1426756 & 233598 & 11800 & 218771 & 3406 & 235463 & 1492 & 1172065 & 0 \\
\hline 56 & 35 & 1426756 & 233598 & 11800 & 218771 & 3406 & 235463 & 1492 & 1172065 & 0 \\
\hline 56 & 35 & 1426756 & 233598 & 11800 & 218771 & 3406 & 235463 & 1492 & 1172065 & 0 \\
\hline 80 & 38 & 233706 & 544706 & 198238 & 8999 & 15313 & 100677 & 1723 & 982389 & 0 \\
\hline 80 & 38 & 233706 & 544706 & 198238 & 8999 & 15313 & 100677 & 1723 & 982389 & 0 \\
\hline 80 & 38 & 233706 & 544706 & 198238 & 8999 & 15313 & 100677 & 1723 & 982389 & 0 \\
\hline 69 & 35 & $121398 \varepsilon$ & 1313389 & 135437 & 26947 & 28576 & 347434 & 11030 & 3722 & 0 \\
\hline 69 & 35 & $121398 \varepsilon$ & 1313389 & 135437 & 26947 & 28576 & 347434 & 11030 & 3722 & 0 \\
\hline 69 & 35 & $121398 \varepsilon$ & 1313389 & 135437 & 26947 & 28576 & 347434 & 11030 & 3722 & 0 \\
\hline 66 & 21 & 14188 & 250782 & 7250 & 38364 & 1773 & 44576 & 1132 & 279271 & 0 \\
\hline 66 & 21 & 14188 & 250782 & 7250 & 38364 & 1773 & 44576 & 1132 & 279271 & 0 \\
\hline 66 & 21 & 14188 & 250782 & 7250 & 38364 & 1773 & 44576 & 1132 & 279271 & 0 \\
\hline 66 & 21 & 1391456 & 1647610 & 30953 & 640673 & 2316 & 329709 & 7576 & 2002589 & 0 \\
\hline
\end{tabular}

Fig 3.0 Meditation and Attention values

\section{wienyou}

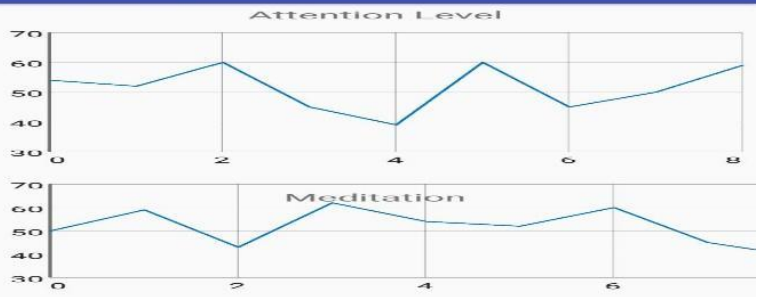

Fig 4.0: Resulting meditation, attention level in App

We also developed a dashboard which shows real-time data. The device can be put up on a person's head and it monitors its attention, concentration, meditation and overall confusion score over a period of time following which it reflects the same on the dashboard.

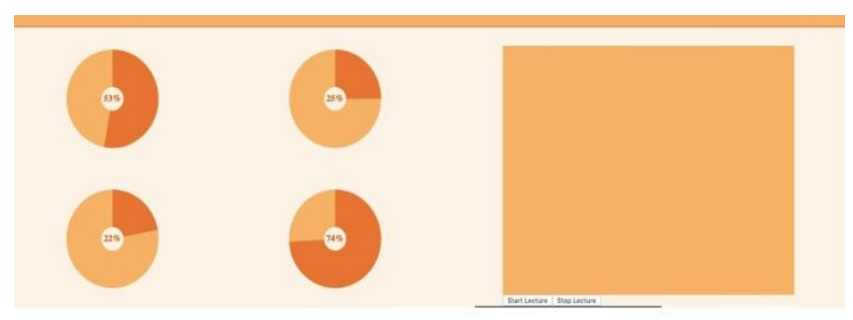

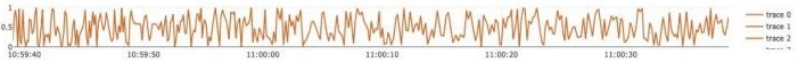

\section{Fig 5.0: Brain Computer Interface Dashboard}

After this we have also developed a heat map, which shows the correlation between various attributes such as the concentration, attention, meditation values etc.

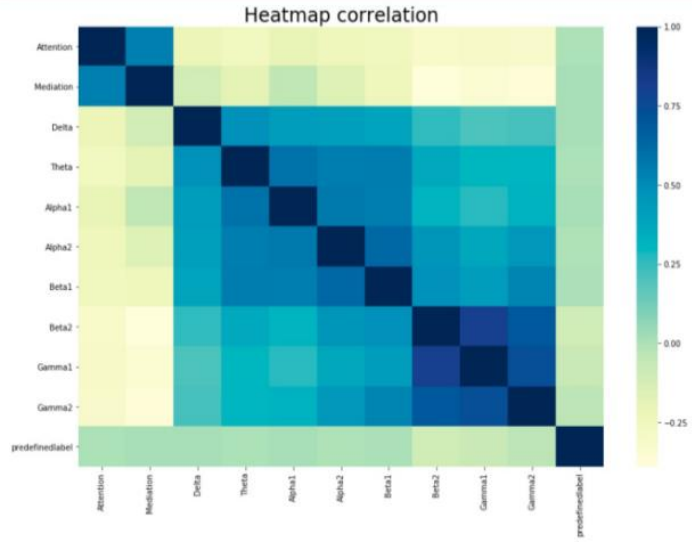

Fig 6.0: Heat-map Correlation

We have also developed the android app for social security in times of emergency with various features to tackle emergency situations and to alert the concerned persons friends and family members.
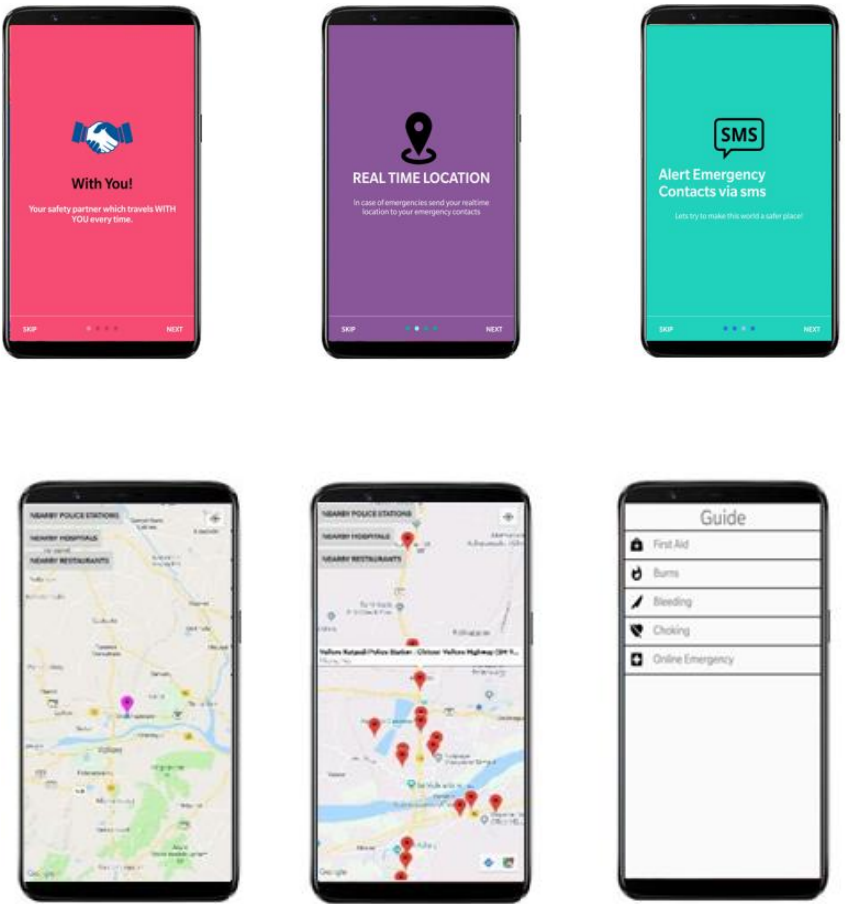

Fig 7.0 The supporting Android Application

We tried out different algorithms for our classification into different brain wave categories and we got the most accurate results from the Random Forest Classifier as shown in the table below.

\begin{tabular}{|c|c|}
\hline Classifier & Accuracy Score \\
\hline KNN & 0.6352 \\
\hline Naive Bayes & 0.6782 \\
\hline XGBoost & 0.7329 \\
\hline Random Forest & 0.9648 \\
\hline
\end{tabular}

Table2: Accuracy scores of different algorithms

Published By:

Blue Eyes Intelligence Engineering \& Sciences Publication 


\section{CONCLUSION}

Mental Health is a very important issue faced by a lot of people in this society. With increasing workloads, social media influence, peer pressure etc, there seems to be a decline in the concentration and meditation levels among people, especially children and teenagers. This can be used in various applications - in classrooms by teachers to monitor weak students, by psychiatrists and councilors for suicide prevention and for mental health analysis of children, teenagers etc. The most important part of this product is our unique algorithm and the interactive brain computer interface which helps to easily calculate a confusion score and get a qualitative measure of mental health. Its availability in the form of a web dashboard as well as an Android Application with its emergency features makes it a product which can be used by people in the daily lives.

\section{REFERENCES}

1. Heath' world organization - OMS. "Suicide, August 2017.Retrived from http://www.who.int/mediacentre/factsheets/fs339/es/consulted.

2. Media centre. (2016, December 19). Retrieved from https://www.who.int/mental_health/advocacy/en/\#Factsheets

3. World Health Organization, Mental health in India. (2017, July 13). Retrieved http://www.searo.who.int/india/topics/mental_health/about_mentalhea lth/en/

4. Suicidio y comportamiento suicida : MedlinePlus enciclopedia médica. (n.d.). Retrieved from https://medlineplus.gov/spanish/ency/article/001554.htm

5. Chiang, W., Cheng, P., Su, M., Chen, H., Wu, S., \& Lin, J. (2011). Socio-health with personal mental health records: Suicidal-tendency observation system on Facebook for Taiwanese adolescents and young adults. 2011 IEEE 13th International Conference on E-Health Networking, Applications and Services. doi:10.1109/health.2011.6026784

6. Calderon-Vilca, H. D., Wun-Rafael, W. I., \& Miranda-Loarte, R. (2017). Simulation of suicide tendency by using machine learning. 2017 36th International Conference of the Chilean Computer Science Society (SCCC). doi:10.1109/sccc.2017.8405128

7. Huang, Z., Dong, M., Mao, Q., \& Zhan, Y. (2014). Speech Emotion Recognition using CNN. 2014 In Proceedings of the 22nd ACM international conference on Multimedia. Florida, US: ACM, 801- 804

8. Liu, Y., Sourina, O., \& Nguyen, M. K. (2011). Real-Time EEG-Based Emotion Recognition and Its Applications. Lecture Notes in Computer Science Transactions on Computational Science XII,256277, vol 6670. Springer, Berlin, Heidelberg. doi:10.1007/978-3-64222336-5_13

9. Ackermann, P., Kohlschein, C., Bitsch, J. A., Wehrle, K., \& Jeschke, S. (2016). EEG-based automatic emotion recognition: Feature extraction, selection and classification methods. 2016 IEEE 18th International Conference on E-Health Networking, Applications and Services (Healthcom). doi:10.1109/healthcom.2016.7749447

10. Berrouiguet, S., Billot, R., Lenca, P., Tanguy, P., Baca-Garcia, E., Simonnet, M., \& Gourvennec, B. (2016). Toward E-Health Applications for Suicide Prevention. 2016 IEEE First International Conference on Connected Health: Applications, Systems and Engineering Technologies (CHASE). doi:10.1109/chase.2016.37

11. G. Lv, S. Hu and X. Lu, "Speech emotion recognition based on dynamic models," 2014 International Conference on Audio, Language and Image Processing, Shanghai, 2014, pp. 480-484. doi: 10.1109/ICALIP.2014.7009840

12. Z. Zhou, H. Jiang and X. Song, "EEG-based emotion recognition using wavelet features," 2014 IEEE 5th International Conference on Software Engineering and Service Science, Beijing, 2014, pp. 585588. doi:10.1109/ICSESS.2014.6933636

13. T. Lv, J. Yan and H. Xu, "An EEG emotion recognition method based on AdaBoost classifier," 2017 Chinese

Automation Congress (CAC), Jinan, 2017, pp. 6050-6054 doi:10.1109/CAC.2017.8243867

14. Teo, J., \& Chia, J. T. (2018). Deep Neural Classifiers For Eeg-Based Emotion Recognition In Immersive Environments. 2018 International
Conference on Smart Computing and Electronic Enterprise (ICSCEE). doi:10.1109/icscee.2018.8538382

15. Cheng, C., Wei, X., \& Jian, Z. (2017). Emotion recognition algorithm based on convolution neural network. 2017 12th International Conference on Intelligent Systems and Knowledge Engineering (ISKE). doi:10.1109/iske.2017.8258786

16. Ghare, P. S., Paithane, A. N., Human Emotion Recognition using Non Linear and Non Stationary EEG Signal, International Conference on Automatic Control and Dynamic Optimization Techniques, 2016, pp. 1013-1016.

17. Y. Gao, H. J. Lee, R. M. Mehmood, "Deep learninig of EEG signals for emotion recognition", Multimedia \& Expo Workshops (ICMEW) 2015 IEEE International Conference on, pp. 1-5, 2015.

18. Lahane, P., \& Thirugnanam, M. (2017). A novel approach for analyzing human emotions based on electroencephalography (EEG). 2017 Innovations in Power and Advanced Computing Technologies (i-PACT). doi:10.1109/ipact.2017.8245056

19. MindWave. (n.d.). Retrieved April 04, 2019, from https://store.neurosky.com/pages/mindwave

\section{AUTHORS PROFILE}

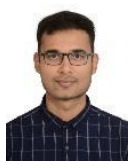

Harsh Kumar, is a final year B. Tech Computer Science student from the School of Computer Science and Engineering at VIT Vellore. His research interests lie in Machine Learning, Brain Computer Interface, Network Optimization and Neural Networks.

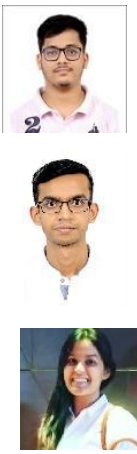

Mayank Sethia, is a final year B. Tech Computer Science student from the School of Computer Science and Engineering at VIT Vellore. His interests lie in Human Computer Interaction and User Interface Design.

Himanshu Thakur, is a final year B. Tech Computer Science student from the School of Computer Science and Engineering at VIT Vellore. His research interests lie in the areas of Deep Learning, Brain Computer Interface and Natural Language Processing.

Ishita Agrawal, is a final year B. Tech Computer Science student from the School of Computer Science and Engineering at VIT Vellore. Her interests lie in Data Science, Statistical Modelling and Linear Algebra.

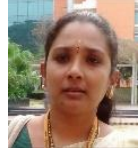

Swarnalatha $\mathbf{P}$, is Associate Professor at the School of Computer Science and Engineering at VIT Vellore. 